\title{
A New Approach to 3D ICP Covariance Estimation
}

\author{
Martin Brossard $^{1}$, Silvère Bonnabel ${ }^{1}$, and Axel Barrau ${ }^{2}$
}

\begin{abstract}
In mobile robotics, scan matching of point clouds using Iterative Closest Point (ICP) allows estimating sensor displacements. It may prove important to assess the associated uncertainty about the obtained rigid transformation, especially for sensor fusion purposes. In this paper we propose a novel approach to 3D uncertainty of ICP that accounts for all the sources of error as listed in Censi's pioneering work [1], namely wrong convergence, underconstrained situations, and sensor noise. Our approach builds on two facts. First, the uncertainty about the ICP's output fully depends on the initialization accuracy. Thus speaking of the covariance of ICP makes sense only in relation to the initialization uncertainty, which generally stems from odometry errors. We capture this using the unscented transform, which also reflects correlations between initial and final uncertainties. Then, assuming white sensor noise leads to overoptimism as ICP is biased owing to e.g. calibration biases, which we account for. Our solution is tested on publicly available real data ranging from structured to unstructured environments, where our algorithm predicts consistent results with actual uncertainty, and compares favorably to previous methods.
\end{abstract}

Index Terms-probability and statistical methods, localization

\section{INTRODUCTION}

$\mathbf{P}$ OINT clouds and the Iterative Closest Point (ICP) algorithm play a crucial role for localization and mapping in modern mobile robotics [2,3]. ICP computes an estimate of the $3 \mathrm{D}$ rigid transformation that aligns a reading point cloud to a reference point cloud (or more generally a model or a surface). The algorithm starts with a first transformation estimate, and repeats - until convergence - point association and least-square minimization, where initialization is naturally provided in mobile robotics by odometry $[4,5]$ based on wheel speeds, inertial sensors, or vision. The point association matches points between the two clouds by generally associating each point of the second cloud to its closest point in the first one. Then, the algorithm minimizes a userchosen metric between the matched points that provides an update of the current estimate. In spite of robust filtering that are broadly used during the alignment of point clouds, a.k.a. registration, ICP is subject to errors stemming from

Manuscript received: September, 10, 2019; Revised November, 1, 2019; Accepted December, 23, 2019.

This paper was recommended for publication by Editor Sven Behnke upon evaluation of the Associate Editor and Reviewers' comments.

${ }^{1}$ Martin Brossard and Silvère Bonnabel are with MINES ParisTech, PSL Research University, Centre for Robotics, 60 Boulevard Saint-Michel, 75006 Paris, France \{martin.brossard, silvere.bonnabel\}@mines-paristech. fr

${ }^{2}$ Axel Barrau is with Safran Tech, Groupe Safran, Rue des Jeunes Bois-Châteaufort, 78772, Magny Les Hameaux Cedex, France axel.barraudsafrangroup.com

Digital Object Identifier (DOI): see top of this page.

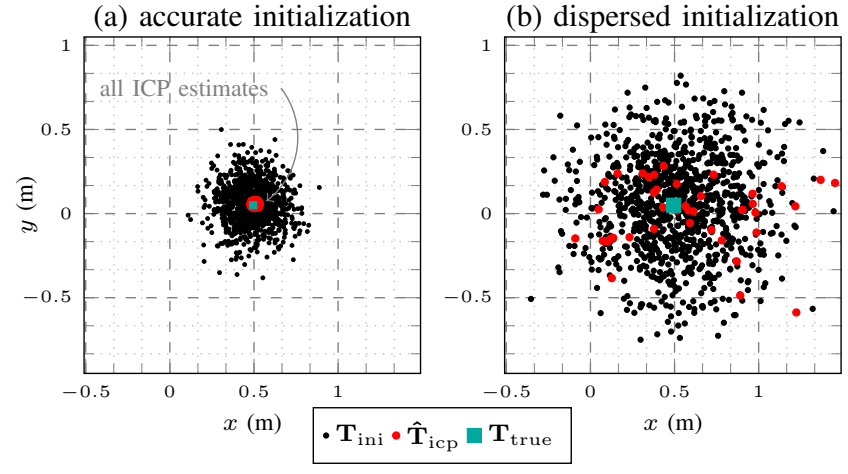

Fig. 1. Horizontal translation estimates according to ICP ( $\hat{\mathbf{T}}_{\mathrm{icp}}$, red dots) for various initial estimates $\left(\mathbf{T}_{\text {ini }}\right.$, black dots) and ground-truth ( $\mathbf{T}_{\text {true }}$, square) for registering two scans of the sequence Stairs of [8], where we sample 1000 initial estimates from two distributions reflecting accurate (a) and dispersed (b) ICP initialization and that respectively correspond to the easy and medium scenarios of [9]. We see the uncertainty on the ICP estimate, that is, dispersion of red points, wholly depends on the accuracy of initialization. There is no "uncertainty of ICP" per se.

sensor noises, underconstrained environments that result in unobservable directions, and local minima $[1,6,7]$.

\section{A. Sources of ICP Uncertainty}

The pioneering work of Censi [1] identifies the following sources of error for ICP registration: wrong convergence (not handled by Censi's formula), underconstrained situations, and sensor noise. As indicated by preliminary remarks in [10,11] we believe a fourth important source is missing: the one that stems from sensor biases. In the present paper we consider indeed the following sources of error:

1) Initial Transformation: ICP is subject to error due to wrong initialization that makes the algorithm converge to a local minimum out of the attraction basin of the true solution, as largely observed in practice, see e.g. $[7,12]$ and Figure 1. In practice it often proves to be the dominant error.

2) Sensor White Noise: each point measured in a point cloud is affected by an independent random sensor noise of centimetric magnitude which is a function of point depth and beam angle $[6,13]$.

3) Sensor Bias Noise: the observed points share common errors that stem from: temperature drift effect, i.e. stability of the laser [13]; observed material [6]; incidence and beam angles resulting in large bias [14]; or wrong calibration, e.g. [15] found a distortion of $0.22 \mathrm{deg}$ of the scan point clouds due to intrinsic calibration process. This correlated noise, a.k.a. bias, strictly limits the confidence we may have in the ICP estimate. To our best knowledge this is often omitted with a few exceptions: e.g., [14] removes bias on point 
measurements due to sensor beam angle, and preliminary ideas may be found in $[10,11]$.

4) Randomness Inherent to the ICP Algorithm: ICP is generally configured with random filtering processes [2], e.g. sub-sampling, such that two solutions with exactly the same inputs would differ.

In the following we address uncertainty coming from 1), 2) and 3) and do not consider 4), which should be marginal.

\section{B. Brief Literature Review}

Various approaches exist for estimating the covariance of the ICP algorithm, each of which being a trade-off between accuracy and execution time. Monte-Carlo algorithms, e.g. $[12,16]$, sample noisy scans (from a reference scan) and ICP initializations to compute a large number of ICP registration results, define the covariance of the sampled results as the covariance estimation, and use the estimated covariance for all future registration with the reference scan, thus getting a covariance function of the reference scan only. Another category of covariance estimation methods relies on closedform expressions [1,17]-[19], whose underlying assumption consists in linearizing the objective function used in ICP around the convergence point, ruling out the possibility for wrong convergence and the uncertainty that stems from it. Albeit still used in practice, Censi's pioneering formula [1] is widely considered as overoptimistic, see e.g. [20]. Recently, [7] leveraged learning based approaches to estimate ICP uncertainty stemming from inaccurate ICP initialization.

\section{Contributions and Paper's Organization}

Our approach introduced in Section II extends existing works in three ways: 1) we consider ICP uncertainty coming both from sensor errors and ICP initialization. 2) we raise an important point which is that ICP uncertainty in itself is meaningless as it is inherently related to uncertainty in the initialization pose (unless there is a global minimum). This is supported by experiments displayed in Figure 1. We address this problem by outputting a covariance matrix of larger dimension that also reflects the correlation between ICP final and initial estimates. And 3) we estimate in Section III the ICP uncertainty combining a closed-form expression using $[1,18]$ accounting for sensor biases, and derivative-free methods using the unscented transform of [21,22], which comes at a lower computational cost than Monte-Carlo runs.

Besides, we evaluate, compare and discuss our approach on the dataset of [8] in Sections IV and V, where our approach obtains consistent estimates and achieves better results than existing methods. The code to reproduce the results of the paper is made publicly available at: https://github. com/CAOR-MINES-ParisTech/3d-icp-cov.

Throughout the article, we configurate the ICP as suggested in [9] with a point-to-plane error metric.

\section{PROPOSED APPROACH}

\section{A. Pose Representation and Pose Uncertainty Representation}

The true transformation between two point clouds and its ICP-based estimate both live in the set of 3D rigid transformations

$$
S E(3):=\left\{\mathbf{T}=\left[\begin{array}{cc}
\mathbf{R} & \mathbf{t} \\
\mathbf{0} & 1
\end{array}\right] \in \mathbb{R}^{4 \times 4} \mid \mathbf{R} \in S O(3), \mathbf{t} \in \mathbb{R}^{3}\right\},
$$

and are thus represented by a matrix $\mathbf{T}$ (a.k.a. homogeneous coordinates), where $\mathbf{R}$ denotes a rotation matrix and $\mathbf{t}$ a translation. Note that, it is consistent with matrix multiplication: if $\mathbf{T}_{1}$ transforms a first point cloud into a second one, and then $\mathbf{T}_{2}$ transforms the latter into a third cloud, then the matrix $\mathbf{T}_{2} \mathbf{T}_{1} \in S E(3)$ encodes the transformation between the first and the third clouds.

It is possible to linearize poses through the approximations $\cos (\alpha) \simeq 1$ and $\sin (\alpha) \simeq \alpha$ for small $\alpha$. For example, we have for a small rotation around the $x$ axis of angle $\alpha$

$$
\begin{aligned}
\mathbf{R}_{x, \alpha} & =\left[\begin{array}{ccc}
1 & 0 & 0 \\
0 & \cos (\alpha) & -\sin (\alpha) \\
0 & \sin (\alpha) & \cos (\alpha)
\end{array}\right] \simeq\left[\begin{array}{ccc}
1 & 0 & 0 \\
0 & 1 & -\alpha \\
0 & \alpha & 1
\end{array}\right] \\
& \simeq \mathbf{I}_{3}+\left[\begin{array}{ccc}
0 & 0 & 0 \\
0 & 0 & -\alpha \\
0 & \alpha & 0
\end{array}\right]=\mathbf{I}_{3}+\alpha\left(\left[\begin{array}{l}
1 \\
0 \\
0
\end{array}\right]\right)_{\times},
\end{aligned}
$$

where $(\mathbf{b})_{\times} \in \mathbb{R}^{3 \times 3}$ denotes the skew symmetric matrix associated with cross product with $\mathbf{b} \in \mathbb{R}^{3}$. Along those lines, a full rotation $\mathbf{R}$ may be approximated as

$$
\mathbf{R} \simeq\left[\begin{array}{ccc}
1 & -\gamma & \beta \\
\gamma & 1 & -\alpha \\
-\beta & \alpha & 1
\end{array}\right]=\mathbf{I}_{3}+\left(\left[\begin{array}{l}
\alpha \\
\beta \\
\gamma
\end{array}\right]\right)_{\times}
$$

for small rotations around the $x, y$ and $z$ axes. The identity pose writes $I d=\mathbf{I}_{4}$ and a transformation being close to identity may thus be linearized as $\mathbf{T} \simeq \mathbf{I}_{4}+\boldsymbol{\xi}^{\wedge}$ with

$$
\boldsymbol{\xi}^{\wedge}:=\left[\begin{array}{cc}
(\phi)_{\times} & \boldsymbol{\rho} \\
\mathbf{0} & 0
\end{array}\right] \in \mathbb{R}^{4 \times 4}, \boldsymbol{\xi}=\left[\begin{array}{l}
\boldsymbol{\phi} \\
\boldsymbol{\rho}
\end{array}\right], \boldsymbol{\phi} \in \mathbb{R}^{3}, \boldsymbol{\rho} \in \mathbb{R}^{3} .
$$

This may serve as an uncertainty representation for poses as follows. If $\boldsymbol{\xi}$ is taken random, typically we take a Gaussian $\boldsymbol{\xi} \sim \mathcal{N}(\mathbf{0}, \mathbf{Q})$, where $\mathbf{Q} \in \mathbb{R}^{6 \times 6}$ is the covariance matrix, then $\mathbf{I}_{4}+\boldsymbol{\xi}^{\wedge}$ defines a small random pose close to identity, i.e., a small transformation. In turn, for a given pose $\mathbf{T}$, the transformation $\hat{\mathbf{T}}=\mathbf{T}\left(\mathbf{I}_{4}+\boldsymbol{\xi}^{\wedge}\right)=\mathbf{T}+\mathbf{T} \boldsymbol{\xi}^{\wedge}$ denotes a random transformation being close to $\mathbf{T}$. $\mathbf{T}$ may be viewed as the noise free mean of the random pose $\hat{\mathbf{T}}$, and $\mathbf{Q}$ encodes the dispersion around the mean value $\mathbf{T}$.

A further theoretical step in this direction consists in using the notion of concentrated Gaussian distribution as advocated in [23], see also [24]-[26],

$$
\hat{\mathbf{T}}=\mathbf{T} \exp (\boldsymbol{\xi}), \text { where } \boldsymbol{\xi} \sim \mathcal{N}(\mathbf{0}, \mathbf{Q}), \boldsymbol{\xi} \in \mathbb{R}^{6},
$$

with $\boldsymbol{\xi}$ a zero-mean Gaussian variable of covariance $\mathbf{Q}$ and where $\exp (\cdot)$ denotes the exponential map of $S E(3)$. The latter maps elements $\boldsymbol{\xi}$ to poses. Albeit sounder from a mathematical standpoint, this is very close to what we have just presented since the $\exp (\cdot)$ map has the property that 
$\exp (\boldsymbol{\xi}) \simeq \mathbf{I}_{4}+\boldsymbol{\xi}^{\wedge}$ up to first order term in $\boldsymbol{\xi}$. For uncertainty representation (4), we adopt the notation $\hat{\mathbf{T}} \sim \mathcal{N}_{L}(\mathbf{T}, \mathbf{Q})$.

Note that, in (4), the vector $\xi \in \mathbb{R}^{6}$ may be viewed as the error between $\mathbf{T}$ and $\hat{\mathbf{T}}$. Indeed the relative transformation between poses $\mathbf{T}$ and $\hat{\mathbf{T}}$ is encoded in $\boldsymbol{\xi}$ as $\mathbf{T}^{-1} \hat{\mathbf{T}}=\exp (\boldsymbol{\xi})$.

\section{B. The Role of ICP Initialization}

The ICP procedure seeks to estimate the transformation $\mathbf{T}_{\text {true }} \in S E(3)$ that maps a first cloud of points $\mathcal{P}$ to a second cloud (or a model) $\mathcal{Q}$ as follows [2,3]:

i) we have a first "guess" for the transformation we call $\mathbf{T}_{\text {ini }}$, a.k.a. initial or coarse alignment [3];

ii) then we initialize the ICP algorithm by applying a transformation $\mathbf{T}_{\mathrm{ini}}^{-1}$ to the cloud $\mathcal{Q}$. This way the transformation the ICP seeks to estimate become the relative pose $\mathbf{T}_{\text {rel }}:=\mathbf{T}_{\text {ini }}^{-1} \mathbf{T}_{\text {true. }}$. We thus get an estimate $\hat{\mathbf{T}}_{\text {rel }}=\operatorname{icp}\left(\mathcal{P}, \mathbf{T}_{\text {ini }}^{-1} \mathcal{Q}\right)$ for $\mathbf{T}_{\text {rel }}$;

iii) finally the estimate of $\mathbf{T}_{\text {true }}$ that the algorithm outputs is $\hat{\mathbf{T}}_{\text {icp }}:=\mathbf{T}_{\text {ini }} \hat{\mathbf{T}}_{\text {rel }}=\mathbf{T}_{\text {ini icp }}\left(\mathcal{P}, \mathbf{T}_{\text {ini }}^{-1} \mathcal{Q}\right)$.

Note that if $\mathbf{T}_{\text {rel }}$ is perfectly estimated we recover $\mathbf{T}_{\text {true }}$ as then $\hat{\mathbf{T}}_{\text {icp }}=\mathbf{T}_{\text {ini }} \mathbf{T}_{\text {rel }}=\mathbf{T}_{\text {ini }} \mathbf{T}_{\text {ini }}^{-1} \mathbf{T}_{\text {true }}=\mathbf{T}_{\text {true }}$ no matter how far the initial guess $\mathbf{T}_{\text {ini }}$ is from $\mathbf{T}_{\text {true }}$.

Let us introduce the various errors at play in ICP. In robotics, the initial guess in $i$ ) is typically provided through inertial sensors or wheeled odometry [4,5]. We have thus an initialization error that stems from sensor imperfections, encoded by a vector $\boldsymbol{\xi}_{\text {ini }}$, and one may write

$$
\mathbf{T}_{\text {ini }}=\mathbf{T}_{\text {true }} \exp \left(\boldsymbol{\xi}_{\text {ini }}\right), \boldsymbol{\xi}_{\text {ini }} \sim \mathcal{N}\left(\mathbf{0}, \mathbf{Q}_{\text {ini }}\right),
$$

which is advocated in $[23,27]$ to suit particularly well represent odometry errors in terms of pose. Then, ICP estimates the relative transformation between $\mathbf{T}_{\text {true }}$ and $\mathbf{T}_{\text {ini }}$, that is, outputs an estimate $\hat{\mathbf{T}}_{\text {rel }}$ of the actual initial error $\mathbf{T}_{\text {rel }}$ which writes

$$
\begin{aligned}
\mathbf{T}_{\text {rel }} & =\mathbf{T}_{\text {ini }}^{-1} \mathbf{T}_{\text {true }}=\exp \left(-\boldsymbol{\xi}_{\text {ini }}\right) \mathbf{T}_{\text {true }}^{-1} \mathbf{T}_{\text {true }} \\
& =\exp \left(-\boldsymbol{\xi}_{\text {ini }}\right) \simeq \mathbf{I}_{4}-\boldsymbol{\xi}_{\text {ini }}^{\wedge} .
\end{aligned}
$$

\section{ICP Estimate $\hat{\mathbf{T}}_{\text {rel }}$ of Relative Pose $\mathbf{T}_{\mathrm{rel}}$}

At step $i$ i) above, that is, once initialization is done, see [2], ICP provides an estimate $\hat{\mathbf{T}}_{\text {rel }}$ of the relative transformation $\mathbf{T}_{\text {rel }}$ of (6) as a function

$$
\hat{\mathbf{T}}_{\text {rel }}:=\operatorname{icp}\left(\mathcal{P}, \mathbf{T}_{\text {ini }}^{-1} \mathcal{Q}\right)
$$

of the point clouds $\mathcal{P}$ and $\mathcal{Q}$. Thus $\hat{\mathbf{T}}_{\text {rel }}$ appears as a function $f\left(\mathbf{T}_{\text {rel }}\right) \in S E(3)$ of the true relative transformation $\mathbf{T}_{\text {rel }}$, typically affected by the phenomena of wrong convergence. Moreover, sensor (scanner) noise induces small fluctuations in the point clouds that affects this estimation. This yields:

$$
\hat{\mathbf{T}}_{\text {rel }}=f\left(\mathbf{T}_{\text {rel }}\right) \exp (\mathbf{G w})=f\left(\exp \left(-\boldsymbol{\xi}_{\text {ini }}\right)\right) \exp (\mathbf{G w}),
$$

where $\mathbf{w} \in \mathbb{R}^{6 K}$ encodes errors due to sensor noise on each of the $K$ pairs of points in the clouds, and $\mathbf{G w} \in \mathbb{R}^{6}$ the resulting 6 degrees of freedom error made on $\mathbf{T}_{\text {rel }}$. Sensor noise stems from unknown parameters that depend upon the i) ICP initialization: $t_{\text {ini }}=t_{\text {true }}+\xi_{\text {ini }}$ [see (5)]

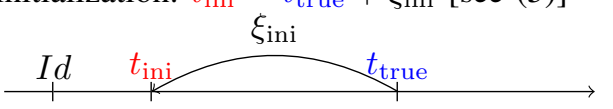

ii) ICP relative pose estimate: $\hat{t}_{\text {rel }}=f\left(t_{\text {true }}-t_{\text {ini }}, \mathbf{w}\right)$

$$
\begin{aligned}
\hat{t}_{\text {rel }} & \simeq I d+\mathbf{J}\left(t_{\text {true }}-t_{\text {ini }}\right)+\mathbf{G w} \\
& \simeq I d-\mathbf{J} \xi_{\text {ini }}+\mathbf{G w}[\text { [see (9)] }
\end{aligned}
$$

iii) ICP final pose estimate: $\hat{t}_{\text {icp }}=t_{\text {ini }}+\hat{t}_{\text {rel }}$ [see (12)]

$$
\begin{aligned}
& \stackrel{I d}{\underbrace{}_{\text {icp }}=\hat{t}_{\text {icp }}-t_{\text {true }}} \\
& \simeq(\mathbf{I}-\mathbf{J}) \xi_{\text {ini }}+\mathbf{G w}
\end{aligned}
$$

Fig. 2. Schematic illustration of the ICP procedure, error definitions and linearizations in the case of $1 \mathrm{D}$ translation $t \in \mathbb{R}(I d=0)$.

calibration process and drift with temperature [13]. If the ICP is initialized on the true pose $\mathbf{T}_{\text {true }}$ then there is no wrong convergence and the only error stems from noise, i.e., $f\left(\exp (\mathbf{0})=f\left(\mathbf{I}_{4}\right)=\mathbf{I}_{4}\right.$. Thus $f(\cdot) \in S E(3)$ is close to $\mathbf{I}_{4}$, and the model may be linearized around $\boldsymbol{\xi}_{\mathrm{ini}}=\mathbf{0}, \mathbf{w}=\mathbf{0}$ as

$$
\begin{aligned}
f\left(\exp \left(-\boldsymbol{\xi}_{\text {ini }}\right)\right) \exp (\mathbf{G w}) & \simeq f\left(\mathbf{I}_{4}-\boldsymbol{\xi}_{\text {ini }}^{\wedge}\right) \exp (\mathbf{G w}) \\
& \simeq \mathbf{I}_{4}+\left(-\mathbf{J} \boldsymbol{\xi}_{\text {ini }}+\mathbf{G w}\right)^{\wedge},
\end{aligned}
$$

where matrix $\mathbf{J}$ encodes the linear approximation of $f(\cdot)$.

\section{ICP Final Pose Error}

Let us now consider step iii) of the ICP algorithm, i.e., the final estimate

$$
\begin{aligned}
\hat{\mathbf{T}}_{\text {icp }} & =\mathbf{T}_{\text {ini }} \hat{\mathbf{T}}_{\text {rel }} \\
& =\mathbf{T}_{\text {true }} \exp \left(\boldsymbol{\xi}_{\text {ini }}\right) f\left(\exp \left(-\boldsymbol{\xi}_{\text {ini }}\right)\right) \exp (\mathbf{G w}) .
\end{aligned}
$$

(11) was obtained substituting (5) and (8) in (10). Linearizing (11) by recalling (9) and keeping only the first order in the small errors $\xi_{\text {ini }}$ and $\mathbf{w}$ yields $\left.\hat{\mathbf{T}}_{\text {icp }} \simeq \mathbf{T}_{\text {true }}\left[\mathbf{I}_{4}+\left(\boldsymbol{\xi}_{\text {ini }}\right)^{\wedge}\right]\left[\mathbf{I}_{4}+\left(-\mathbf{J} \boldsymbol{\xi}_{\text {ini }}+\mathbf{G w}\right)\right)^{\wedge}\right] \simeq$ $\mathbf{T}_{\text {true }}\left(\mathbf{I}_{4}+\left(\boldsymbol{\xi}_{\text {ini }}-\mathbf{J} \boldsymbol{\xi}_{\text {ini }}+\mathbf{G w}\right)^{\wedge}\right)$ and thus in terms of uncertainty representation (4) we approximately find:

$$
\hat{\mathbf{T}}_{\text {icp }} \simeq \mathbf{T}_{\text {true }} \exp \left(\left(\mathbf{I}_{6}-\mathbf{J}\right) \boldsymbol{\xi}_{\text {ini }}+\mathbf{G w}\right) .
$$

Figure 2 recaps the computations for 1D translations. There are a couple of situations of interest. Let us momentarily assume sensor noise to be turned off, $\mathbf{w}=\mathbf{0}$, for simplicity.

- If there is one global minimum, then the ICP systematically recovers the relative transformation (6) at step $i i)$ of the algorithm, i.e. $\hat{\mathbf{T}}_{\text {rel }}=\mathbf{T}_{\text {rel }}$ and thus $f\left(\mathbf{T}_{\text {rel }}\right)=\mathbf{T}_{\text {rel }}$. So $f\left(\exp \left(-\boldsymbol{\xi}_{\text {ini }}\right)\right) \simeq \mathbf{I}_{4}-\boldsymbol{\xi}_{\text {ini }}^{\wedge}$ and we identify $\mathbf{J}=\mathbf{I}_{6}$ in this case. As a result the final estimate (12) is $\mathbf{T}_{\text {true }} \exp (\mathbf{0})=\mathbf{T}_{\text {true }}$ indeed.

- On the other hand, in the directions where we have no information, e.g. along hallways or in underconstrained environment [1], the relative transformation will not be 
affected in the corresponding directions meaning that (along those directions) $\mathbf{J}=\mathbf{0}$ and the final error then has the form $\mathbf{T}_{\text {true }}^{-1} \hat{\mathbf{T}}_{\text {icp }}=\mathbf{T}_{\text {true }}^{-1} \mathbf{T}_{\text {true }} \exp \left(\boldsymbol{\xi}_{\text {ini }}\right)=$ $\exp \left(\boldsymbol{\xi}_{\text {ini }}\right)$, that is, the initialization error fully remains.

In intermediate cases (when there are local minima) the remaining error is a fraction $\mathbf{J} \boldsymbol{\xi}_{\text {ini }}$ of the initialization error.

\section{E. Corresponding ICP Error Covariance}

If we represent ICP uncertainties resorting to concentrated Gaussian (4) as $\hat{\mathbf{T}}_{\text {icp }} \sim \mathcal{N}_{L}\left(\mathbf{T}_{\text {true }}, \mathbf{Q}_{\text {icp }}\right)$, i.e., we posit $\mathbf{T}_{\text {true }}^{-1} \hat{\mathbf{T}}_{\mathrm{icp}}=\exp \left(\boldsymbol{\xi}_{\mathrm{icp}}\right)$, then the covariance matrix $\mathbf{Q}_{\text {icp }}$ of $\boldsymbol{\xi}_{\text {icp }}$ describes dispersion (hence uncertainty) of the ICP error. Plugging the latter representation into (12), we have $\boldsymbol{\xi}_{\text {icp }}=\left(\mathbf{I}_{6}-\mathbf{J}\right) \boldsymbol{\xi}_{\text {ini }}+\mathbf{G w}$. As the initialization error is assumed to have covariance matrix $\mathbf{Q}_{\text {ini }}$ typically inferred though an odometry error model $[23,27]$ and by denoting $\mathbf{Q}_{\text {sensor }}$ the covariance of scan sensor noise $\mathbf{w}$, the covariances add up owing to independence of sensor noises, and by squaring $\boldsymbol{\xi}_{\text {icp }}=\left(\mathbf{I}_{6}-\mathbf{J}\right) \boldsymbol{\xi}_{\text {ini }}+\mathbf{G w}$ we find

$$
\mathbf{Q}_{\text {icp }}=\left(\mathbf{I}_{6}-\mathbf{J}\right) \mathbf{Q}_{\text {ini }}\left(\mathbf{I}_{6}-\mathbf{J}\right)^{T}+\mathbf{G Q}_{\text {sensor }} \mathbf{G}^{T} \text {. }
$$

This is our first result about ICP covariance. The first term related to the initialization uncertainty and that accounts for wrong convergence, lack of constraints in the clouds and unobservable directions, and the second one related to scan noise and that may be computed through "Censi-like" [1] formulas as we will show in section III.

\section{F. Discussion}

Albeit not obvious, $\mathbf{J}$ actually heavily depends on $\mathbf{Q}_{\text {ini }}$. This is an insight of the present paper: uncertainty of ICP does not exist in itself. Assume indeed there are various local minima. If $\mathbf{Q}_{\text {ini }}$ is very small, then all initializations $\mathbf{T}_{\text {ini }}$ fall within the attraction basin of $\mathbf{T}_{\text {true }}$ and thus $f\left(\mathbf{T}_{\text {rel }}\right)=\mathbf{T}_{\text {rel }}$ and we identify $\mathbf{J}=\mathbf{I}_{6}$. But if $\mathbf{Q}_{\text {ini }}$ is large enough only a fraction of initializations $\mathbf{T}_{\text {ini }}$ lead to $f\left(\mathbf{T}_{\text {rel }}\right)=\mathbf{T}_{\text {rel }}$, the ones that get trapped in other local minimas do not lead to correct estimate of $\mathbf{T}_{\text {rel }}$ and $\mathbf{J} \neq \mathbf{I}_{6}$. Thus $\mathbf{J}$ is not the analytical Jacobian of function $f(\cdot)$, and may be viewed as its "statistical linearization" [28]. This prompts the use of an unscented transform [21] to compute it, see Section III-B.

\section{G. Maximum Likelihood Fusion of Initial and ICP Estimates}

$\mathbf{T}_{\text {ini }}$ and $\hat{\mathbf{T}}_{\text {icp }}$ may be viewed as two estimates of $\mathbf{T}_{\text {true }}$ associated with uncertainty respectively $\mathbf{Q}_{\text {ini }}=\operatorname{cov}\left(\boldsymbol{\xi}_{\text {ini }}\right)$ and $\mathbf{Q}_{\text {icp }}=\operatorname{cov}\left(\boldsymbol{\xi}_{\text {icp }}\right)$ where $\boldsymbol{\xi}_{\text {icp }}=(\mathbf{I}-\mathbf{J}) \boldsymbol{\xi}_{\text {ini }}+\mathbf{G w}$. The corresponding pose fusion problem of finding the Maximum Likelihood (ML) of a pose $\mathbf{T}_{\text {true }}$ given two uncertain pose estimates was considered in [23], with the important difference that herein $\boldsymbol{\xi}_{\text {ini }}$ and $\boldsymbol{\xi}_{\text {icp }}$ are not independent, they are correlated, with joint matrix of initialization and ICP errors

$$
\mathbf{Q}:=\operatorname{cov}\left(\left[\begin{array}{l}
\boldsymbol{\xi}_{\text {ini }} \\
\boldsymbol{\xi}_{\text {icp }}
\end{array}\right]\right)=\left[\begin{array}{cc}
\mathbf{Q}_{\text {ini }} & \mathbf{Q}_{\text {ini }}(\mathbf{I}-\mathbf{J})^{T} \\
(\mathbf{I}-\mathbf{J}) \mathbf{Q}_{\text {ini }} & \mathbf{Q}_{\text {icp }}
\end{array}\right] .
$$

Using linearization as previously and following first-order computations in [23], the maximum likelihood estimate of $\mathbf{T}_{\text {true }}$ may be approximated as $\hat{\mathbf{T}}_{\mathrm{ML}}=\mathbf{T}_{\text {true }} \exp \left(\boldsymbol{\xi}_{\mathrm{ML}}\right)$ with $\operatorname{cov}\left(\boldsymbol{\xi}_{\mathrm{ML}}\right)=\mathbf{Q}_{\mathrm{ML}}$, with $\mathbf{Q}_{\mathrm{ML}}$ defined through its inverse:

$$
\mathbf{Q}_{\mathrm{ML}}^{-1}=\left[\begin{array}{l}
\mathbf{I} \\
\mathbf{I}
\end{array}\right]^{T}\left[\begin{array}{cc}
\mathbf{Q}_{\text {ini }} & \mathbf{Q}_{\text {ini }}(\mathbf{I}-\mathbf{J})^{T} \\
(\mathbf{I}-\mathbf{J}) \mathbf{Q}_{\text {ini }} & \mathbf{Q}_{\text {icp }}
\end{array}\right]^{-1}\left[\begin{array}{l}
\mathbf{I} \\
\mathbf{I}
\end{array}\right] \text {. }
$$

The latter stems from classical linear estimation theory and may be proved using the Kalman information filter: to the first order $\hat{\mathbf{T}}_{\text {ini }}$ and $\hat{\mathbf{T}}_{\text {icp }}$ are considered as two noisy measurements of $\mathbf{T}_{\text {true }}$ with joint covariance (14), the measurement matrix is thus $\mathbf{H}:=\left[\begin{array}{ll}\mathbf{I} & \mathbf{I}\end{array}\right]^{T}$ and as $\mathbf{T}_{\text {true }}$ is initially totally unknown the prior covariance satisfies $\mathbf{P}^{-1}=\mathbf{0}$. The covariance of the Kalman estimate in the light of measurements is thus updated in information form as $\mathbf{P}^{-1} \leftarrow \mathbf{0}+\mathbf{H}^{T} \mathbf{Q}^{-1} \mathbf{H}$.

\section{Practical Covariance Computation}

This section describes our algorithm for estimating the 3D ICP uncertainty covariance (13) leveraging findings of Section II. We propose to first compute the rightmost term of (13) which is due to sensor noise.

\section{A. Computation of Dispersion Owing to Sensor Noise}

We now focus on the computation of $\mathbf{G Q}_{\text {sensor }} \mathbf{G}^{T}$. The cost function of point-to-plane ICP after initialization writes $J_{\hat{\mathbf{T}}_{\text {rel }}}\left(\mathcal{P}, \mathbf{T}_{\text {ini }}^{-1} \mathcal{Q}\right)=\sum_{k=1}^{K}\left\|\left(\hat{\mathbf{T}}_{\text {rel }} p_{k}-\tilde{q}_{k}\right) \cdot n_{k}\right\|^{2}$, where the $\tilde{q}_{k}$ 's denote the points of $\mathbf{T}_{\text {ini }}^{-1} \mathcal{Q}$ and $K$ is the number of pairs of matched points. Linearizing on $S E(3)$, we may linearize the cost $J_{\hat{\mathbf{T}}_{\text {rel }} \exp (\boldsymbol{\xi})}\left(\mathcal{P}, \mathbf{T}_{\text {ini }}^{-1} \mathcal{Q}\right)=\sum_{k=1}^{K} \|\left(\hat{\mathbf{T}}_{\text {rel }} \exp (\boldsymbol{\xi}) p_{k}-\right.$ $\left.\tilde{q}_{k}\right) \cdot n_{k} \|^{2}$ w.r.t. estimate $\hat{\mathbf{T}}_{\text {rel }}$ at $\hat{\mathbf{T}}_{\text {rel }}=\mathbf{T}_{\text {rel }}$ as

$$
J_{\hat{\mathbf{T}}_{\text {rel }} \exp (\boldsymbol{\xi})}\left(\mathcal{P}, \mathbf{T}_{\text {ini }}^{-1} \mathcal{Q}\right) \simeq \sum_{k=1}^{K}\left\|\mathbf{B}_{k} \boldsymbol{\xi}-d_{k}\right\|^{2},
$$

with $d_{k}$ a scalar being function of differences between pairs of points and point normals. Least squares formulas yield an optimal value $\boldsymbol{\xi}^{*}=\mathbf{A}^{-1} \sum_{k=1}^{K} \mathbf{B}_{k}^{T} d_{k}$, where we let $\mathbf{A}=$ $\sum_{k=1}^{K} \mathbf{B}_{k}^{T} \mathbf{B}_{k}$. Each $d_{k}$ is affected by $k$-th component $w_{i}$ of previously introduced sensor noise $\mathbf{w}$, and this induces fluctuations in $\boldsymbol{\xi}^{*}$ over various experiments. Let's postulate $w_{i}=\mathbf{b}+\nu_{i}$ with $\nu_{i}$ a white noise of variance $\sigma^{2}$, and $\mathbf{b}$ and unknown calibration bias that is identical for all points but varies from one experiment to the next. Following least squares covariance, see $[1,18]$, we end up with:

$$
\mathbf{G Q}_{\text {sensor }} \mathbf{G}^{T}=\sigma^{2} \mathbf{A}^{-1}+\mathbf{A}^{-1} \mathbf{B} \operatorname{cov}(\mathbf{b}) \mathbf{B}^{T} \mathbf{A}^{-1},
$$

where $\mathbf{A}=\sum_{k=1}^{K} \mathbf{B}_{k}^{T} \mathbf{B}_{k}$, and $\mathbf{B}=\sum_{k=1}^{K} \mathbf{B}_{k}^{T}$. We recover the covariance $\sigma^{2} \mathbf{A}^{-1}$ of $[1,18]$ w.r.t. sensor white noise, and a new term, $\mathbf{A}^{-1} \mathbf{B} \operatorname{cov}(\mathbf{b}) \mathbf{B}^{T} \mathbf{A}^{-1}$, that represents the covariance w.r.t. the unknown bias $\mathbf{b}$, that is, correlated noise. This new additional term is paramount as $\mathbf{A}$ has magnitude proportional to $K$, hence $\mathbf{A}^{-1}$ is very small, explaining that Censi's formula (based on $\mathbf{A}^{-1}$ only) seems overoptimistic [20]. For example $\mathbf{A}^{-1}$ has trace $0.2 \mathrm{~cm}$ for the registration displayed in Figure 1 whereas the covariance 


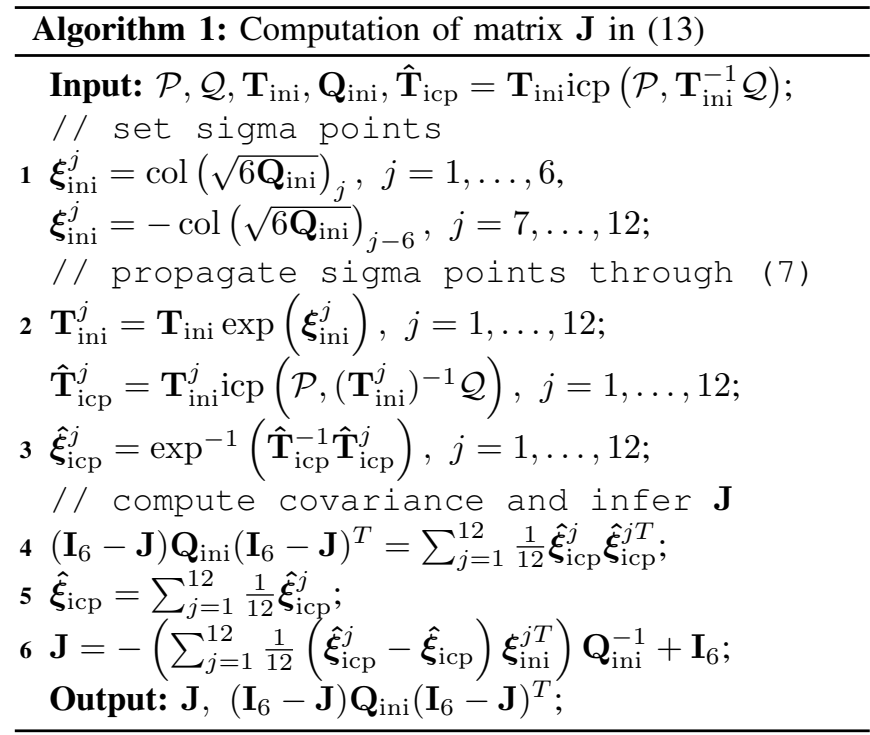

w.r.t. the unknown bias has trace $2.6 \mathrm{~cm}$. In practice $\mathbf{b}$ arises from sensor calibration, laser stability [13], observed material [6], and incidence of beams [14]. In the remainder we assume bias standard deviation to be approximately $5 \mathrm{~cm}$ as in [6].

Note that (17) captures the effect of underconstrained situations like hallways. Indeed in unobservable directions the cost $J_{\hat{\mathbf{T}}_{\text {rel }}}(\cdot)$ is constant, yielding small eigenvalues for $\mathbf{A}$ and hence large covariance (17). Derivation of $\mathbf{B}_{k}$ and extraction of $\mathbf{G}$ in (17) are available with paper code.

\section{B. Computation of Dispersion owing to ICP Initialization}

Computation of $\left(\mathbf{I}_{6}-\mathbf{J}\right) \mathbf{Q}_{\text {ini }}\left(\mathbf{I}_{6}-\mathbf{J}\right)^{T}$ in (13) is of greater importance as in practice it largely dominates $\mathbf{G Q}_{\text {sensor }} \mathbf{G}^{T}$. We propose to compute it in a deterministic derivative-free method, in which we adapt the unscented transform [21] for pose by following $[22,23]$. The advantages of using our unscented based method rather than Monte-Carlo sampling are fourfold: 1) it is deterministic; 2) it remains computationally reasonable by adding only 12 ICP registrations; 3 ) it explicitly computes the cross-covariance matrix between $\hat{\mathbf{T}}_{\mathrm{icp}}$ and $\mathbf{T}_{\text {ini }}$ as a by-product without extra computational operations; and 4) it scales with $\mathbf{Q}_{\text {ini }}$, i.e. our approach naturally selfadapts to the confidence we have in initialization without extra parameter tuning.

We compute the covariance as follows, see Algorithm 1:

- we consider the prior distribution $\mathbf{T}_{\text {prior }} \sim$ $\mathcal{N}_{L}\left(\mathbf{T}_{\text {ini }}, \mathbf{Q}_{\text {ini }}\right)$, which is approximated by a set of so-called sigma-points $\boldsymbol{\xi}_{\text {ini, }}^{j}$, see step 1);

- we approximate the propagated distribution $\mathbf{T}_{\mathrm{prop}}=$ $\mathbf{T}_{\text {prior }} \operatorname{icp}\left(\mathcal{P}, \mathbf{T}_{\text {prior }}^{-1} \mathcal{Q}\right)$ as

$$
\begin{aligned}
\mathbf{T}_{\text {prop }} & =\mathcal{N}_{L}\left(\mathbf{T}_{\text {ini }}, \mathbf{Q}_{\text {ini }}\right) \operatorname{icp}\left(\mathcal{P}, \mathcal{N}_{L}\left(\mathbf{T}_{\text {ini }}, \mathbf{Q}_{\text {ini }}\right)^{-1} \mathcal{Q}\right) \\
& \sim \mathcal{N}_{L}\left(\hat{\mathbf{T}}_{\text {icp }},\left(\mathbf{I}_{6}-\mathbf{J}\right) \mathbf{Q}_{\text {ini }}\left(\mathbf{I}_{6}-\mathbf{J}\right)^{T}\right),
\end{aligned}
$$

after propagating each sigma-point in steps 2) and 3), where $\hat{\mathbf{T}}_{\text {icp }}$ is the given ICP pose estimate. We compute

\begin{tabular}{c||c|c|c|c|c||c|c|c|c|c}
\hline \multirow{2}{*}{ metric } & \multicolumn{2}{|c|}{ NNE } & \multicolumn{2}{c|}{ KL div. } & \multicolumn{2}{c|}{ NNE* $^{*}$} & \multicolumn{2}{|c}{ KL div.* } \\
& trans. & rot. & trans. & rot. & trans. & rot. & trans. & rot. \\
\hline$\hat{\mathbf{Q}}_{\text {censi }}$ & $10^{3}$ & $10^{3}$ & $10^{4}$ & $10^{5}$ & 38 & $10^{2}$ & $10^{3}$ & $10^{5}$ \\
$\hat{\mathbf{Q}}_{\text {carlo }}^{\text {monte }}$ & $10^{3}$ & $10^{2}$ & $10^{4}$ & $10^{4}$ & 22 & 20 & $10^{3}$ & $10^{3}$ \\
proposed & $\mathbf{4 . 2}$ & $\mathbf{3 4}$ & $\mathbf{1 0}^{\mathbf{2}}$ & $\mathbf{1 0}^{\mathbf{2}}$ & $\mathbf{0 . 8}$ & $\mathbf{3 . 8}$ & $\mathbf{3 1}$ & $\mathbf{9 8}$ \\
\hline
\end{tabular}

Table 1. Results of ICP uncertainty estimation in term of Normalized Norm Error (NNE) and Kullbach-Leibler divergence (KL div.) divided into translation and rotation parts. As the ICP error distributions are not Gaussian [9], we provide robust statistics (starred, *) by removing both the more and less accurate quantiles of each registration. The proposed method outperforms the two others.

the covariance and infer the matrix $\mathbf{J}$ as a by-product in respectively steps 4 ) and 6).

We derive the algorithm by following [22] for pose measurement, zero-mean prior distribution, and where we set $\alpha=1$.

\section{EXPERIMENTAL RESULTS}

\section{A. Dataset Description \& ICP Algorithm Setting}

This section evaluates the ability of the approach to estimate ICP uncertainty on the Challenging data sets for point cloud registration algorithms [8]. It comprises eight sequences where point clouds are taken in environments ranging from structured to unstructured, and indoor to outdoor. Each sequence contains between 31 and 45 point cloud scans along with ground-truth pose for each scan, that provides a total of 268 scans and 1020 different registrations as we align each scan with the three scans the following.

We configure the ICP as in [9] with 95\% random subsampling, kd-tree for data association, and point-to-plane error metric where we keep the $70 \%$ closest point associations for rejecting outliers.

\section{B. Compared Methods and Evaluation Metrics}

This section evaluates the following methods:

$\hat{\mathbf{Q}}_{\text {censi }}$ : the close-form method of [1] adapted for the ICP setting defined above;

$\hat{\mathbf{Q}}_{\text {carlo }}^{\text {monte }}$ : the covariance computed after sampling of 65

Monte-Carlo ICP estimates;

$\hat{\mathrm{Q}}_{\text {icp }}$ : our proposed approach detailed in Section III.

Each method assumes depth sensor white noise and bias with $5 \mathrm{~cm}$ standard deviation, which is the mean value found in [6] for the Hokuyo sensor used for these experiments, and all methods know the initial uncertainty $\mathbf{Q}_{\text {ini }}$, whose magnitude $0.1 \mathrm{~m}$ and $10 \mathrm{deg}$ corresponds to the easy scenario of [9].

We compare the above methods using two metrics:

1) Normalized Norm Error (NNE): that evaluates the historically challenging $[1,16]$ prediction of the covariance scale, and is computed as

$$
\mathrm{NNE}=\left(\frac{1}{N} \sum_{n=1}^{N}\left\|\boldsymbol{\xi}_{n}\right\|_{2}^{2} / \operatorname{trace}\left(\hat{\mathbf{Q}}_{n}\right)\right)^{1 / 2},
$$

where $\boldsymbol{\xi}_{n}=\exp ^{-1}\left(\mathbf{T}_{\text {true }}^{-1} \hat{\mathbf{T}}_{n}\right)$ with is the transformation error and $\hat{\mathbf{Q}}_{n}$ the estimated uncertainty covariance matrix, and averaged over $N$ samples. This metric characterizes the 


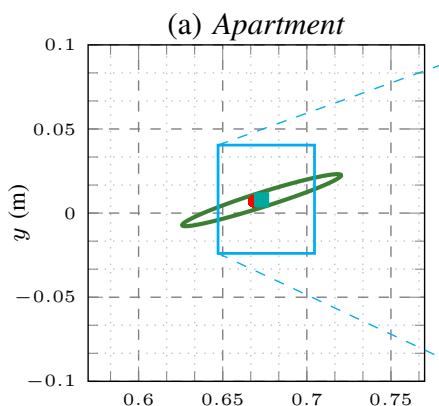

(c) Hauptgebaude

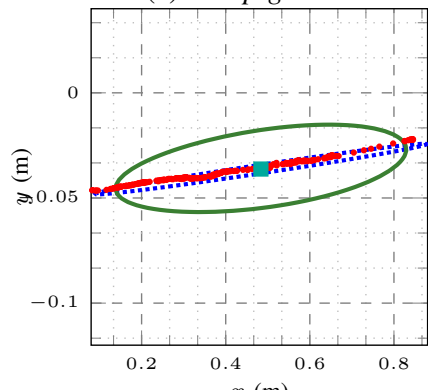

$x(\mathrm{~m})$

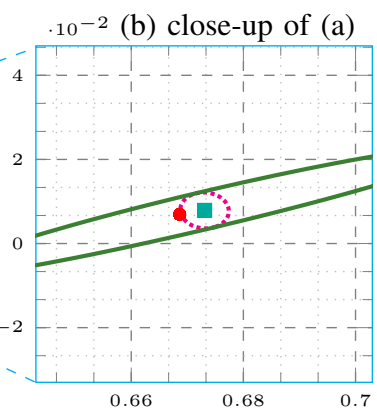

(d) Mountain

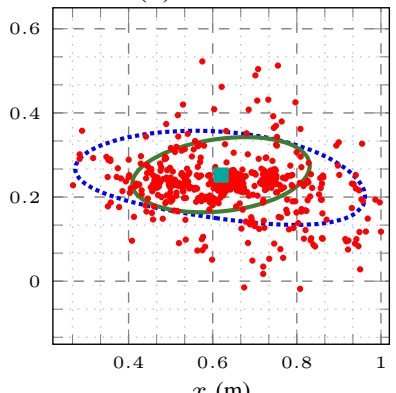

$x(\mathrm{~m})$ $\square \mathbf{T}_{\text {true }} \bullet \hat{\mathbf{T}}_{\mathrm{icp}} \cdots \cdots \cdot \hat{\mathbf{Q}}_{\mathrm{censi}} \cdots \cdots \cdot \cdot \hat{\mathbf{Q}}_{\mathrm{carlo}}^{\text {monte }}-$ proposed

Fig. 3. Results on real data of [8] projected onto the ground plane for visualization. Ellipses represent the $95 \%(3 \sigma)$ confidence sets for each uncertainty estimation method. (a): "true convergence situation", the errors are mainly caused by sensor noises and Censi's formula should apply. (b): however we see the Censi ellipse seems optimistic as ground truth is almost outside it whereas it falls well within our ellipse (green). (c, d): "wrong convergence", the large errors are due ICP initialization. Only our approach is consistent with ICP uncertainty in each environment and does not suffer from overoptimism.

uncertainty as only the true registration is known (the exact distribution of the point cloud is unknown). The target value is one, below one the estimation is pessimistic, whereas a value over one indicates an overoptimistic estimation.

2) Kullback-Leibler Divergence (KL div.): which is computed between a pseudo-true distribution and the estimated distribution. The pseudo-true distribution is computed after sampling 1000 ICP estimates of the evaluated registration over the initial position. As sensor noise is fixed in the point clouds, this distribution represents the uncertainty stemming from initialization errors.

\section{Results}

Results are averaged over 1000 initializations for each of the 1020 considered pairs of point clouds, representing a total of more than one million registrations, where the ICP is initialized with a different estimate $\mathbf{T}_{\text {ini }}$ sampled from $\mathcal{N}_{L}\left(\mathbf{T}_{\text {true }}, \mathbf{Q}_{\text {ini }}\right)$. Table 1 provides average results over the eight sequences, and Figure 3 illustrates typical registrations from structured to unstructured environments. We observe:

- $\hat{\mathbf{Q}}_{\text {censi }}$ is far too optimistic and unreliable for sensorfusion, as noted in [20]. Its centimetric confidence interval makes sense only when ICP is very accurate;

- $\hat{\mathbf{Q}}_{\text {carlo }}^{\text {monte }}$ is overoptimistic when the discrepancy arising from ICP initialization remains negligible, see Figure 3 (b), for which the method predicts a confidence interval with millimetric size. This is naturally explained as the method assumes no error caused by sensor noises;

- the proposed method obtains the best results for both metrics as displayed in Table 1. It notably outperforms $\hat{\mathbf{Q}}_{\text {carlo }}^{\text {monte }}$ while deterministic hence more reliable, and computationally much cheaper. The dominant term is generally due to initial uncertainty. However in "global minimum" cases the sensor bias used for computing $\hat{\mathbf{Q}}_{\text {icp }}$ slightly inflates the covariance of [1], and more closely captures actual uncertainty, see Figure $3(a, b)$.

Besides outperforming the other methods, our method provides simple parameter tuning: we set the bias noise standard deviation as having same magnitude as sensor white noise, and the error stemming from ICP initialization does not need to be tuned when $\mathbf{Q}_{\text {ini }}$ is an output of inertial, visual, or wheeled odometry system $[4,5]$.

Regarding computational complexity and execution time, step 2) of the algorithm requires 12 registrations which take $6 \mathrm{~s}$ when registrations are computed parallely, whereas the remaining part of the algorithm takes less than $0.1 \mathrm{~s}$. The 65 Monte-Carlo runs are more than five times more demanding than the the proposed method.

\section{COMPLEMENTARY EXPERIMENTAL Results}

This section provides an in-depth analysis of the method in term of trajectory consistency, robustness to high and misknown initial uncertainty, and discusses the advantages and the validity of the approach.

\section{A. Application to Trajectory Consistency}

We asses the quality of the covariance estimation in Section III over trajectories as follows. For each sequence of [8], we compute the global pose estimate at scan $l$ by compounding transformations such that $\hat{\mathbf{T}}^{l}=\hat{\mathbf{T}}^{0,1} \ldots \hat{\mathbf{T}}^{l-1, l}$, whose covariance is computed with the closed-form expressions of [23] which are valid up to 4-th order approximation. We compare three methods defined as:

CELLO-3D : reproduced results of [7], that proposes a learning based method for estimating the ICP covariance, which is trained on environments similar to the tested sequence. The results are indicative as the ICP setting of [7] slightly differs from the setting of [9] we use;

ini.+ICP : combines initialization and ICP measurements with the covariance estimate (15) without considering cross-covariance terms, i.e., applying formulas of [23] ; proposed : based on the full proposed covariance of the maximum-likelihood estimate (15).

We set initial errors as in Section IV and evaluate the above methods using the Mahalanobis Distance proposed in [7] between final trajectory estimates and ground truth

$$
\text { Mah. dist. }=\left(\sum_{n=0}^{N} \frac{\boldsymbol{\xi}_{n}^{T} \hat{\mathbf{Q}}_{n}^{-1} \boldsymbol{\xi}_{n}}{\operatorname{dim}\left(\boldsymbol{\xi}_{n}\right) N}\right)^{1 / 2},
$$

where $\boldsymbol{\xi}_{n}=\exp ^{-1}\left(\mathbf{T}_{\text {true }}^{-1} \hat{\mathbf{T}}_{n}\right)$ is the transformation error and $\hat{\mathbf{Q}}_{n}$ the estimated covariance matrix, averaged over $N$ 


\begin{tabular}{|c|c|c|c|c|c|c|c|c|c|c|c|c|c|c|c|c|}
\hline \multirow{2}{*}{$\begin{array}{l}\text { sequence } \\
\text { Mah. dist. }\end{array}$} & \multicolumn{2}{|c|}{ Apartment } & \multicolumn{2}{|c|}{ Hauptgebaude } & \multicolumn{2}{|c|}{ Stairs } & \multicolumn{2}{|c|}{$\| \quad$ Mountain } & \multicolumn{2}{|c|}{ Gazebo summer } & \multicolumn{2}{|c|}{ Gazebo winter } & \multicolumn{2}{|c|}{ Wood summer } & \multicolumn{2}{|c|}{ Wood winter } \\
\hline & trans. & rot. & trans. & rot. & trans. & rot. & || trans. & rot. & trans. & rot. & trans. & rot. & trans. & rot. & trans. & rot. \\
\hline & & & & & & & - & - & & & & & & & & 0.3 \\
\hline & & 1 & & 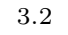 & & & 1. & 1. & & & & & & & & 4.8 \\
\hline proposed & 2.3 & 9.8 & 1.8 & 2.9 & 1.1 & 4.2 & 1.2 & 1.2 & 1.0 & 2.3 & 1.8 & 3.7 & 1.5 & 4.7 & 1.2 & 4.2 \\
\hline
\end{tabular}

Table 2. Trajectory consistency results in term of Mahalanobis distance (bold indicates best performance) split into translation and rotation parts for the sequences of [8], where Mountain is not considered in [7]. Our method obtains on average the best uncertainty assessment, albeit slightly optimistic.

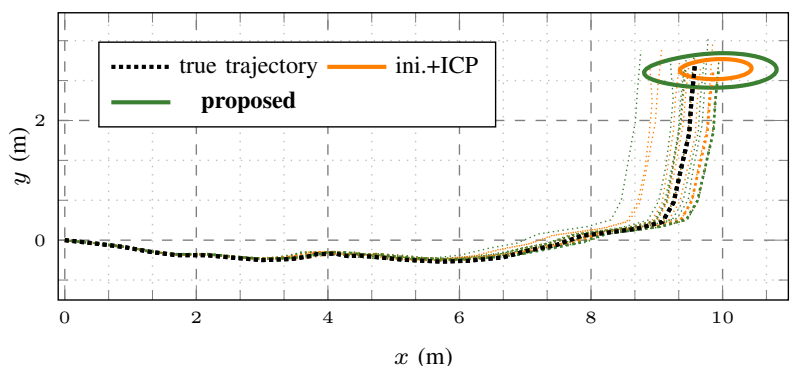

Fig. 4. Results projected onto the ground plane for visualization in the

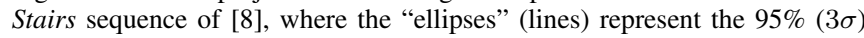
final confidence sets.

samples. The target value is one, below one the estimation is pessimistic, and above one the estimates are optimistic.

We average results over 40 different initial trajectories for each sequence, which are numerically displayed in Table 2 and illustrated in Figure 4. We observe:

- CELLO-3D is the only pessimistic method, which estimates uncertainty ranging from 3 to 10 times higher than actual uncertainty. It evidences how difficult it is to asses ICP uncertainty in practice;

- the proposed approach obtains on average the best results. It obtains similar estimates than ini.+ICP when the ICP algorithm is accurate (Gazebo and Wood). In more difficult environments, e.g. Stairs, it better incorporates initialization than ini.+ICP thanks to it accounting for measurement correlation encoded in (14), see Figure 4.

These results confirm the ability of the method to compute covariance estimates over trajectories also and the relevance of correlation terms between ICP and initial estimates.

\section{B. Role of Initial Uncertainties in Covariance Estimation}

We evaluate the influence of $\mathbf{Q}_{\mathrm{ini}}$ on the covariance estimation in challenging situations where $\mathbf{Q}_{\text {ini }}$ is high, inaccurately known (the estimation of $\mathbf{Q}_{\text {ini }}$ is in itself challenging), and sensor noise is inflated. For each environment of [8], we evaluate the method in 9 situations where initial uncertainty is easy, medium and difficult with respectively $0.1,0.5,1 \mathrm{~m}$ and $10,20,50 \mathrm{deg}$ standard deviation, see [9]. In each situation we evaluate the algorithm with different magnitudes for $\mathbf{Q}_{\text {ini }}$, hence assessing its robustness to pessimistic and optimisitic parametrization. We finally add white and depth bias noises on already noisy point clouds with $5 \mathrm{~cm}$ standard deviation. Results are given in Table 3, and illustrated in Figure 5.

- The ICP algorithm obtains unreliable results for large initial uncertainty, see Figure 5, whereas it obtains centimetric errors for low levels of initial uncertainty. As anticipated in Section II-B, significant ICP errors are caused by inaccurate initialization;

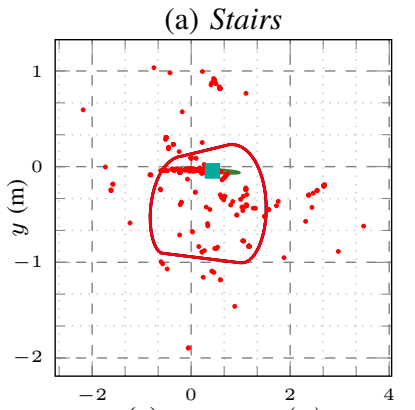

(c) same as (a)

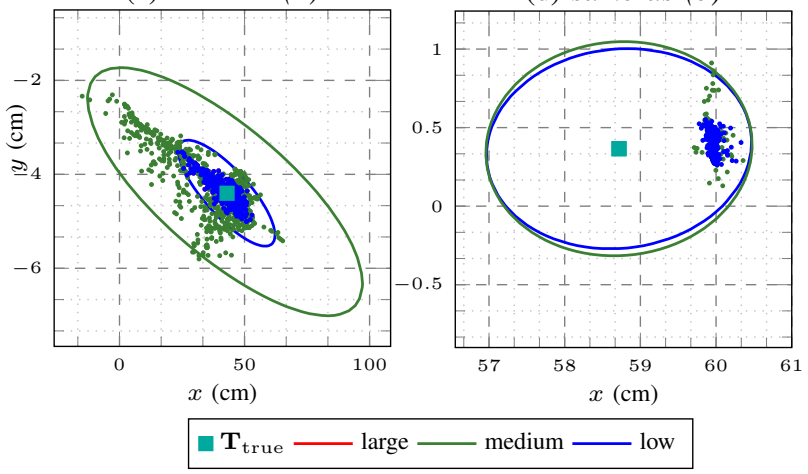

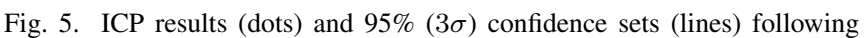
our method for three levels of initial uncertainty. We see the latter highly influences the ICP registrations and ICP covariance estimations accordingly.

- ICP final outputs are agnostic to initialization when a global minimum exists, see e.g. the Gazebo and Wood environments in Table 3 for levels of $\mathbf{Q}_{\mathrm{ini}}$ corresponding to easy and medium scenario. The method obtains correct estimates where the sensor noise terms numerically dominate the estimated covariances, and $\mathbf{J} \approx \mathbf{I}$, see Section II-D;

- another environments contain local minima, e.g. Hauptgebaude. Then the algorithm outputs reflect the pessimistic or optimistic belief about the initial uncertainty. We recommend in these situations to set $\mathbf{Q}_{\text {ini }}$ sufficiently high to favour conservatism;

- our method is able to detect inaccurate ICP registrations by providing very high covariance estimates, although it cannot accurately describe non Gaussian distributions, see Figure $5(a, b)$.

\section{Discussion about the Proposed Approach}

We finally examine the pros, cons, and fundamental assumptions of the method. The main advantages are: it being anchored in a mathematical theory, its efficiency to assess uncertainty for acceptable levels of initial uncertainties, 


\begin{tabular}{c||c|c|c|c|c|c|c|c|c|c}
\hline $\mathbf{Q}_{\text {ini }}$ (true) & \multicolumn{3}{c|}{ easy } & \multicolumn{3}{c|}{ medium } & \multicolumn{3}{c}{ difficult } \\
\hline$\hat{\mathbf{Q}}_{\text {ini }}$ (algo) & || & easy & med. & diff. & easy & med. & diff. & easy & med. & diff. \\
\hline Appart. & 1.6 & 1.5 & $10^{-6}$ & 1.8 & 1.7 & $10^{-6}$ & $10^{5}$ & $10^{5}$ & 0.6 \\
Haupt. & 1.8 & 0.3 & $10^{-3}$ & 3.6 & 0.7 & $10^{-3}$ & $10^{3}$ & $10^{3}$ & 0.3 \\
Stairs & 0.7 & 0.2 & $10^{-3}$ & 1.9 & 0.8 & $10^{-3}$ & $10^{3}$ & $10^{3}$ & 0.7 \\
Montain & 2.1 & 1.1 & $10^{-3}$ & 3.3 & 1.8 & $10^{-3}$ & $10^{3}$ & $10^{3}$ & 4.0 \\
Gazebo & 1.1 & 1.0 & $10^{-5}$ & 1.3 & 1.2 & $10^{-5}$ & $10^{4}$ & $10^{4}$ & 0.8 \\
Wood & 2.1 & 2.1 & $10^{-3}$ & 2.2 & 2.2 & $10^{-3}$ & $10^{3}$ & $10^{3}$ & 0.3 \\
\hline
\end{tabular}

Table 3. NNE, see (19), for different levels of true and supposed initial uncertainty. Difficult initial uncertainty leads to highly erroneous ICP outputs that the proposed method detects if correctly parametrized.

its simplicity, while being computationally reasonable, see Algorithm 1. The cross-covariance term in (15) may be fruitful for increasing robustness of back-end systems, e.g. pose-graph [20], as it correlates two previously supposed independent measurements. Comparisons between diagonal terms in (15) finally provides a way for trading-off between initial odometry guesses and ICP estimates, see [29].

The Gaussian error assumption of the ICP estimates is the core hypothesis of the method. We required this assumption to obtain a tractable method able to provide a covariance for a state estimator, e.g. a Kalman filter. However, if one pursues a more accurate estimation of the ICP distribution, we suggest massive sampling methods as an expansive alternative, although our method largely proves sufficient to detect problematic situations.

The method finally requires the covariance of the initial uncertainty as an input. If the provided initialization confidence is inexact, the method outputs may reflect the initial optimism or pessimism in general situations. Nonetheless an insight of the present paper, see Section II and e.g. Figure 5, is that ICP errors intrinsically depend on the initial accuracy so that a coarse idea of initial uncertainty is essential, whatever the method one desires to use.

Finally, [23,26,27] show that concentrated Gaussian distribution (4) faithfully describe robot odometry models, such that methods like the Kalman filter are able to provide an accurate (concentrated) Gaussian approximation to the true initial uncertainty for which our approach has been designed.

\section{CONCLUSION}

This paper presents a novel method for real time estimation of 3D uncertainty covariance matrix of the ICP algorithm. The method relies on a careful study of the influence of both sensor noises and algorithm initialization on the ICP estimates, that we leverage in a deterministic scheme which remains very simple in terms of parameter tuning. The core of our approach is versatile as one can apply it to various choices of error metrics. However with point-to-point ICP the closed form part of the covariance is not valid, see [18]. The approach is successfully validated on individual pairs of point clouds and over trajectories on challenging real datasets, where it obtains consistent results. Future work will address the benefit of the method for preventing ICP failures, particularly its coupling with learning-based methods, and for fusing odometry, ICP and GNSS in Kalman filtering and optimization-based schemes.

\section{REFERENCES}

[1] A. Censi, "An Accurate Closed-form Estimate of ICP's Covariance," in ICRA, 2007, pp. 3167-3172.

[2] F. Pomerleau, F. Colas, and R. Siegwart, "A Review of Point Cloud Registration Algorithms for Mobile Robotics," Found. Trends in Robotics, vol. 4, no. 1, pp. 1-104, 2015.

[3] D. Holz, A. E. Ichim, F. Tombari et al., "Registration with the Point Cloud Library," IEEE RAM, vol. 22, no. 4, pp. 110-124, 2015.

[4] R. Dube, A. Gawel, H. Sommer et al., "An Online Multi-robot SLAM System for 3D LiDARs," in IROS, 2017, pp. 1004-1011.

[5] P. Geneva, K. Eckenhoff, Y. Yang et al., "LIPS: LiDAR-Inertial 3D Plane SLAM," in IROS, 2018, pp. 123-130.

[6] F. Pomerleau, A. Breitenmoser, M. Liu et al., "Noise Characterization of Depth Sensors for Surface Inspections," in CARPI, 2012, pp. 16-21.

[7] D. Landry, F. Pomerleau, and P. Giguère, "CELLO-3D: Estimating the Covariance of ICP in the Real World," in ICRA, 2019.

[8] F. Pomerleau, M. Liu, F. Colas et al., "Challenging Data Sets for Point Cloud Registration Algorithms," IJRR, vol. 31, no. 14, pp. 1705-1711, 2012.

[9] F. Pomerleau, F. Colas, R. Siegwart et al., "Comparing ICP Variants on Real-world Data Sets: Open-source Library and Experimental Protocol," Auton. Robots, vol. 34, no. 3, pp. 133-148, 2013.

[10] S. T. Pfister, K. L. Kriechbaum, S. I. Roumeliotis et al., "Weighted Range Sensor Matching Algorithms for Mobile Robot Displacement Estimation," in ICRA, 2002, pp. 1667-1674.

[11] M. Barczyk and S. Bonnabel, "Towards Realistic Covariance Estimation of ICP-based Kinect V1 Scan Matching: The 1D Case," in ACC, 2017, pp. 4833-4838.

[12] T. M. Iversen, A. G. Buch, and D. Kraft, "Prediction of ICP Pose Uncertainties Using Monte Carlo Simulation with Synthetic Depth Images," in IROS, 2017, pp. 4640-4647.

[13] Z. Wang, Y. Liu, Q. Liao et al., "Characterization of a RS-LiDAR for 3D Perception," in CYBER, 2018.

[14] J. Laconte, S.-P. Deschênes, M. Labussière et al., "LiDAR Measurement Bias Estimation via Return Waveform Modelling in a Context of 3D Mapping," in ICRA, 2019.

[15] J.-E. Deschaud, "IMLS-SLAM: Scan-to-Model Matching Based on 3D Data," in ICRA, 2018, pp. 2480-2485.

[16] O. Bengtsson and A.-J. Baerveldt, "Robot Localization Based on Scanmatching," Robotics and Auto. Sys., vol. 44, no. 1, pp. 29-40, 2003.

[17] P. Biber and W. Strasser, "The Normal Distributions Transform: a New Approach to Laser Scan Matching," in IROS, 2003, pp. 2743-2748.

[18] S. Bonnabel, M. Barczyk, and F. Goulette, "On the Covariance of ICP-based Scan-matching Techniques," in ACC, 2016, pp. 5498-5503.

[19] S. M. Prakhya, L. Bingbing, Y. Rui et al., "A Closed-form Estimate of 3D ICP Covariance," in MVA, 2015, pp. 526-529.

[20] E. Mendes, P. Koch, and S. Lacroix, "ICP-based Pose-graph SLAM," in SSRR, 2016, pp. 195-200.

[21] S. Julier, J. Uhlmann, and H. Durrant-Whyte, "A New Method for the Nonlinear Transformation of Means and Covariances in Filters and Estimators," IEEE T-AC, vol. 45, no. 3, pp. 477-482, 2000.

[22] M. Brossard, S. Bonnabel, and J.-P. Condomines, "Unscented Kalman filtering on Lie groups," in IROS, 2017, pp. 2485-2491.

[23] T. Barfoot and P. Furgale, "Associating Uncertainty With ThreeDimensional Poses for Use in Estimation Problems," IEEE T-RO, vol. 30, no. 3, pp. 679-693, 2014.

[24] A. Barrau and S. Bonnabel, "Invariant Kalman Filtering," Ann. Rev. of Cont., Rob., and Auto. Sys., vol. 1, no. 1, pp. 237-257, 2018.

[25] _ , "The Invariant Extended Kalman Filter as a Stable Observer," IEEE T-AC, vol. 62, no. 4, pp. 1797-1812, 2017.

[26] G. Bourmaud, R. Mégret, M. Arnaudon et al., "Continuous-Discrete Extended Kalman Filter on Matrix Lie Groups Using Concentrated Gaussian Distributions," J Math Imaging Vis, vol. 51, no. 1, pp. 209228, 2015.

[27] A. W. Long, K. C. Wolfe, M. J. Mashner et al., "The banana distribution is gaussian: A localization study with exponential coordinates," Robotics: Science and Systems VIII, vol. 265, 2013.

[28] F. Gustafsson and G. Hendeby, "Some Relations Between Extended and Unscented Kalman Filters," IEEE T-SP, vol. 60, no. 2, pp. 545$555,2012$.

[29] G. Ovchinnikov, A. L. Pavlov, and D. Tsetserukou, "Windowed Multiscan Optimization Using Weighted Least Squares for Improving Localization Accuracy of Mobile Robots," Autonomous Robots, vol. 43, no. 3, pp. 727-739, 2019. 